\title{
Influence of Grain Refinement on Slurry Formation and Surface Segregation in Semi-solid Al-7Si-0.3Mg Castings
}

\author{
JORGE SANTOS, LOTHAR H. KALLIEN, ANDERS E.W. JARFORS, \\ and ARNE K. DAHLE
}

\begin{abstract}
This study aims to evaluate the effect of grain refinement on slurry formation and surface segregation in semi-solid castings produced by the Rheometal ${ }^{\mathrm{TM}}$ process. The effect of two grain refiners, Al-8B and Al-5Ti-1B, on the slurry $\alpha$-Al grain size, shape factor and solid fraction was evaluated. The results suggest that the addition of a grain refiner can affect the solid fraction obtained in the Rheometal ${ }^{\mathrm{TM}}$ process and, consequently, reduce the solute content near the casting surface. Grain refiner addition resulted in a larger fraction of $\alpha$-Al grains $\leq 60 \mu \mathrm{m}$ for the refined alloys compared with the unrefined alloy. Additionally, the growth of $\alpha$-Al slurry globules was greater for the unrefined alloy compared with the refined alloy during solidification in the die-cavity. A more homogeneous and finer microstructure was observed near the surface in the grain-refined castings compared with the unrefined castings. Evidence of significant liquid penetration was identified in some $\alpha$-Al globules, indicating that disintegration of $\alpha$-Al globules may occur during the Rheometal ${ }^{\mathrm{TM}}$ casting process.
\end{abstract}

https://doi.org/10.1007/s11661-018-4787-9

(C) The Author(s) 2018

\section{INTRODUCTION}

GRAIN refinement is commonly used in the aluminium casting industry. ${ }^{[1]}$ Smaller and more globular crystals form in grain-refined aluminium alloys, which results in enhanced feeding, a decreased hot tearing tendency and more uniform dispersion of porosity and intermetallic phases. ${ }^{[2,3]}$ Additionally, improved mechanical properties in the casting, fatigue resistance and increased uniformity of the anodized surface are obtained in grain-refined aluminium alloys. ${ }^{[4]}$ There is divergence in the literature regarding the effects of stirring on the grain refiner effectiveness. Yang et al. ${ }^{[5]}$ concluded that the grain refinement effect of Al-Ti-B additions was lost after electromagnetic stirring of an A356 aluminium alloy. Sharma ${ }^{[6]}$ found a reduction in size and increased globularity of primary $\alpha$-Al obtained by inoculation of the liquid prior to stirring in the Rapid Slurry Forming (RSF) process. In the New Rheocasting (NRC) process, a superheated liquid alloy is poured into

JORGE SANTOS, ANDERS E.W. JARFORS, and ARNE K. DAHLE are with the Department of Materials and Manufacturing, School of Engineering, Jönköping University, P.O. Box 1026, 55111 Jönköping, Sweden. Contact e-mail: jorge.santos@ju.se LOTHAR H. KALLIEN is with the Department of Applied Sciences, Aalen University, Beethovenstrasse 1, 73430 Aalen, Germany.

Manuscript submitted January 26, 2018.

Article published online July 9, 2018 a chill cup to generate copious nucleation of crystals. Easton et al. ${ }^{[7]}$ reported that the addition of a grain refiner did not significantly change the microstructure at low pouring temperatures in the NRC process.

A surface segregation layer is a typical feature in High Pressure Die Casting (HPDC) ${ }^{[8]}$ direct-chill casting ${ }^{[9]}$ and, particularly, Semi-Solid Metal (SSM) processing. ${ }^{109}$ Surface segregation involves a solute-enriched region at the casting surface, with a distinct microstructure compared with the center of the casting and consequently strongly contributes to heterogeneous properties along the cross-section. ${ }^{[8]}$ Gourlay et al. ${ }^{[11]}$ studied defect bands and surface segregation layer formation in HPDC and suggested, in addition to the migration of the externally solidified crystals to the center, that the origin of the segregation layer is a combined effect of inverse segregation and exudation. In both segregation modes, a solute-enriched liquid flows toward the casting surface through the mushy zone to compensate for solidification shrinkage. ${ }^{[1]}$ Exudation occurs when the partially solidified alloy next to the die wall shrinks because of solidification shrinkage and thermal contraction, and a gap is formed between the solid alloy and die wall. Consequently, a pressure differential is formed between the gap and the interior of the solidifying alloy, and the solute-enriched liquid is forced to flow through inter-crystal channels into the space between the solidifying alloy and die wall ${ }^{[11]}$ and solidifies into an almost fully eutectic microstructure. ${ }^{[8]}$ 
In inverse segregation, the solute-enriched liquid that advanced toward the casting surface to compensate for solidification shrinkage solidifies into very small primary $\alpha-\mathrm{Al}$ dendrites and eutectic near the casting surface. ${ }^{[8]}$

In this work, the effect of grain refiner addition on the slurry $\alpha$-Al grain size, shape factor and solid fraction is evaluated. Additionally, the surface segregation region composition and microstructure are analyzed and associated with the effect of grain refiner addition on the primary $\alpha$-Al. Finally, deformation and disintegration of the primary $\alpha$-Al globules during the Rheometal ${ }^{\mathrm{TM}}$ process are investigated.

\section{EXPERIMENTAL}

\section{A. Casting}

Aluminum Al-7Si-0.3 Mg alloy melts were prepared in an electrical resistance furnace, one with the base alloy, one with the addition of $0.15 \mathrm{wt}$ pct Al-8B in the form of a tablet and another with the addition of a 0.20 wt pet A1-5Ti-1B master alloy rod. The master alloy rod and tablet were wrapped in aluminum foil, preheated to $200{ }^{\circ} \mathrm{C}$ and immersed within a preheated ladle into the liquid held at $700{ }^{\circ} \mathrm{C}$. Fifteen minutes after addition, the liquid was stirred for homogenization. The chemical compositions of the Al-7Si-0.3Mg alloys are shown in Table I.

The Rheometal ${ }^{\mathrm{TM}}{ }^{[12]}$ process was used to produce SSM castings from the alloys in Table I. For the slurry preparation, Enthalpy Exchange Material (EEM) was cast from the alloys in a copper die with a 40-mm-diameter cylindrical cavity and internal water-cooling channels. A 12-mm-diameter stainless-steel rod was inserted along the copper die-cavity centerline to be cast in the EEM interior. After casting, the EEMs were cut to have the specific height to produce an addition of 7 pct of the shot weight. Later, the rod and EEM, preheated to $200{ }^{\circ} \mathrm{C}$, were inserted into a stirring device a few seconds before immersion into the liquid. Around $1.3 \mathrm{~kg}$ of superheated aluminum alloy was ladled from the furnace. As the temperature reached $650{ }^{\circ} \mathrm{C}\left(\sim 35^{\circ} \mathrm{C}\right.$ superheat), the preheated EEM was immersed into the liquid while rotating at $850 \mathrm{rpm}$. When the turbulence on the surface stopped, the slurry preparation process was assumed to be complete. The time for preparation was approximately 18 seconds. Subsequently, the prepared slurry was poured into the shot sleeve of a 50-ton Vertical High Pressure Die Casting (VHPDC) machine to produce $10-\mathrm{mm}$-thick tensile bars, shown in Figure 1. The machine parameters were kept constant for all experiments, with a plunger advance speed of $0.3 \mathrm{~m} / \mathrm{s}$ and an intensification pressure of 160 bar. The die temperature was controlled by internal oil circulation set at a constant temperature of $175^{\circ} \mathrm{C}$ using a PolyTemp HTF 300 heater. To maintain reproducible thermal conditions in the shot sleeve and die-cavity, a first set of shots was performed before the experiments started to warm up the assembly. The relevant dimensions of the casting are shown in Figure 1(a). An HPDC casting was produced from the base alloy as reference.

\section{B. Microstructural Characterization}

Longitudinal cross-sections of the castings, as highlighted in Figure 1(b), were studied by metallography. The samples for optical microscopy were ground, and the last step of polishing was completed with a $1-\mu \mathrm{m}$ diamond suspension. A 10 pet $\mathrm{NaOH}$ solution was used to etch the sample surface before microscopy. For Scanning Electron Microscopy (SEM), a last step of polishing with an OP-U suspension was performed before observation.

The manual point count from ASTM E562-11 ${ }^{[13]}$ was followed for the measurements of the solid fraction on micrographs obtained from polished surfaces etched with Weck's reagent $\left(4 \mathrm{~g} \mathrm{KMnO}_{4}, 1 \mathrm{~g} \mathrm{NaOH}\right.$ and $100 \mathrm{~mL}$ distilled water), with a contact time of $12 \mathrm{sec}-$ onds. Gao et al. ${ }^{[14]}$ analyzed the coloring mechanism of the Weck's reagent in A356 alloy specimens quenched in water from the semisolid range. A manganese oxide film formed on the aluminum sample, which was responsible for a brown color with varying brightnesses in the $\alpha$-Al globules observed in the optical microscope. The different brightnesses observed are mainly associated with the varying roughness of the interface formed between the manganese oxide film and the sample surface. Electron probe microanalysis and roughness measurements in the $\alpha$-Al globules showed that in regions where silicon segregated, a smooth interface is formed between the manganese oxide film and the $\alpha$-Al globules. ${ }^{[14]}$ The smooth interface results in a brighter area observed in the periphery of the $\alpha-\mathrm{Al}$ globules compared with the darker color in the core of the $\alpha$-Al globules (where the interface between the manganese oxide film and the substrate have greater roughness). In a further study, Gao et al. ${ }^{[15]}$ measured the solid fraction of a titanium-containing A356 aluminum alloy that was heated and isothermally held at a pre-determined temperature in the semi-solid range. After quenching of the isothermally held samples, the solid fraction was evaluated both from micrographs of as-polished samples and etched with Weck's reagent. The results were compared with calculations by the lever rule from the binary Al-Si phase diagram. The results showed a close agreement between the lever rule prediction and the solid fraction measured on the surface etched with Weck's reagent, while the solid fraction from the as-polished samples was much higher. Other studies have used Weck's reagent to identify the growth layer formed during quenching of semi-solid castings. ${ }^{[16]}$ In this study, the peripheral brighter regions on the $\alpha$-Al globules revealed in micrographs of surfaces etched with Weck's reagent are assumed to have formed during growth of the slurry $\alpha$-Al globules in the die-cavity.

In this work, two populations of grains were distinguished in the microstructures. The $\alpha$-Al globules observed in the microstructure that likely nucleated and grew during slurry preparation and in the shot sleeve were identified as $\alpha_{1}$. Payandeh et al. ${ }^{[17]}$ showed that externally solidified $\alpha$-Al globules of quenched slurries and castings with varied silicon contents had an average diameter $\geq 25 \mu \mathrm{m}$. Therefore, the smaller $\alpha$-Al observed in the microstructure in this study, $<25 \mu \mathrm{m}$ in 
Table I. Compositions of the Al-7Si-0.3Mg Unrefined and Refined Alloys

\begin{tabular}{lccccccrr}
\hline Alloy & $\mathrm{Si}$ & $\mathrm{Fe}$ & $\mathrm{Cu}$ & $\mathrm{Mn}$ & $\mathrm{Mg}$ & $\mathrm{Ti}$ & $\mathrm{Sr}$ & $\mathrm{Al}$ \\
\hline Base & 6.97 & 0.13 & 0.011 & 0.012 & 0.29 & 0.090 & 0.032 & balance \\
Base + Al-8B & 7.08 & 0.15 & 0.017 & 0.011 & 0.30 & 0.070 & 0.012 & balance \\
Base + Al-5Ti-1B & 6.85 & 0.15 & 0.015 & 0.014 & 0.26 & 0.13 & 0.005 & balance \\
\hline \multicolumn{2}{l}{ Composition in weight percent. } \\
\hline
\end{tabular}

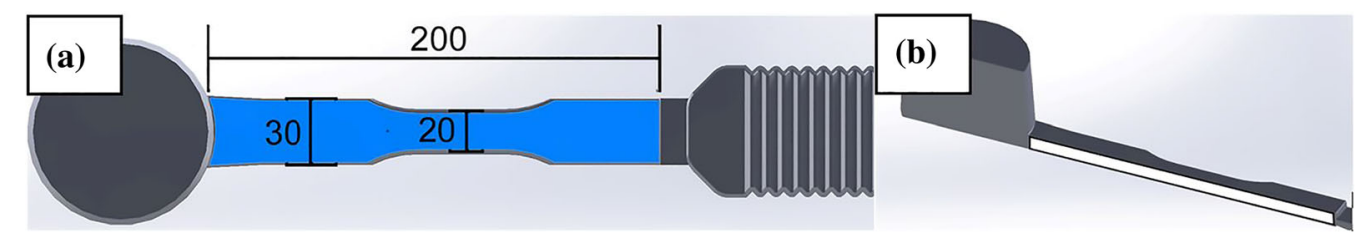

Fig. 1-(a) Casting shape and dimensions in $\mathrm{mm},(b)$ side view of the casting with the longitudinal cross-section investigated highlighted.

diameter, were identified as in-cavity solidified grains. When the distinction of the different $\alpha$-Al in the SSM casting is irrelevant, the general denotation $\alpha$-Al is used.

A JEOL JSM-7001F scanning electron microscope (SEM) equipped with Wavelength-Dispersive Spectrometry (WDS) was used to measure the silicon concentration in the interior of the $\alpha_{1}-\mathrm{Al}$ globules, and pure silicon was used as standard. An area of $225 \mu \mathrm{m}^{2}$ in the interior of ten $\alpha_{1}$-Al globules in each casting was measured with a fixed acceleration voltage of $10 \mathrm{kV}$.

Energy Dispersive X-ray Spectroscopy (EDS) was used to measure the composition of the surface segregation layer. The measurement was performed with a fixed acceleration voltage of $15 \mathrm{kV}$ in ten different regions of each sample. EBSD analysis was performed in the SEM operating at $20 \mathrm{kV}$, with a step size of $5 \mu \mathrm{m}$, to identify the $\alpha$-Al grains (grain boundaries $>15 \mathrm{deg}$ ). The EBSD analysis was performed in the center of the cross-sections, where the Externally Solidified Crystals (ESCs) migrate during die filling. ${ }^{[18]}$ The goal was to analyze the $\alpha_{1}-\mathrm{Al}$ grains. Image analysis software was used to determine the grain size and shape factor of the grains identified by EBSD. The reported values of the grain size and shape factor are the average of at least 150 grains.

\section{RESULTS}

\section{A. Grain Size}

Figure 2 shows EBSD maps where the individual grains are distinguished by different colors. Regions with a difference in crystallographic orientation exceeding $15 \mathrm{deg}$ were defined as different grains, identical to other studies. ${ }^{[19]}$ Additionally, large dendrites were observed in all the alloys, highlighted by circles in Figure 2. These are likely $\alpha$-Al crystals formed in the shot sleeve or on the EEM surface during slurry preparation, denoted freeze-on layer. ${ }^{[20]}$ In the rheo-die-casting process, previous shearing of the slurry $\leq 15$ seconds was found to decrease the volume fraction of $\alpha$-Al crystals formed in the shot sleeve. ${ }^{[21]}$ Additionally, a low superheat results in coarser and more globular crystals formed in the shot sleeve in HPDC. ${ }^{[22]}$ In this work, the previous shearing of the slurry may result in the formation of very few and near-globular crystals in the shot sleeve. Payandeh et al. ${ }^{[23]}$ observed that the freeze-on layer microstructure is composed of long and bent columnar dendrites. Disintegration and growth of the freeze-on layer dendrites occur during the Rheometal $^{\mathrm{TM}}$ process and cavity filling, and the initial size and shape of the crystals are changed. Therefore, the large dendrites observed in Figure 2 most likely originate from the freeze-on layer.

To investigate the effect of grain refinement on the size and shape of the $\alpha_{1}$-Al grains, the average equivalent circular diameter and shape factor of the $\alpha-\mathrm{Al}$ grains were determined for all the alloys. The very small grains (diameter $\leq 25 \mu \mathrm{m}$ ) and the large dendrites (diameter $\geq 190 \mu \mathrm{m}$ ) were not considered because grain refinement probably is not a determinant factor for these grain size populations. The results are shown in Table II as the average equivalent circular diameter and shape factor. The average equivalent circular diameter is the diameter of a circle with the same area as the $\alpha-\mathrm{Al}$ grain. The shape factor is the grain area relative to the area of a circle with the same perimeter. The results show that the average equivalent grain diameter and shape factor are similar for all alloys. These results are not in agreement with other studies where grain refinement reduced the grain size and increased the sphericity of grains in SSM castings. ${ }^{[24-26]}$

The $\alpha$-Al grains (Figure 2 ) were grouped in equivalent circular diameter ranges, and the cumulative distribution function was obtained. The cumulative distribution function shows the probability of grain size less than or equal to a specific equivalent circular diameter. The results are shown in Figure 3 as a cumulative distribution function as a function of the equivalent circular grain diameter. Data obtained from the HPDC casting are also plotted for comparison. The HPDC casting showed the greatest probability of obtaining $\alpha$-Al grains with an equivalent circular diameter up to $60 \mu \mathrm{m}$ compared with the SSM castings, as seen in Figure 3. In the grain-refined castings there is greater probability to obtain primary $\alpha$-Al grains with an equivalent 

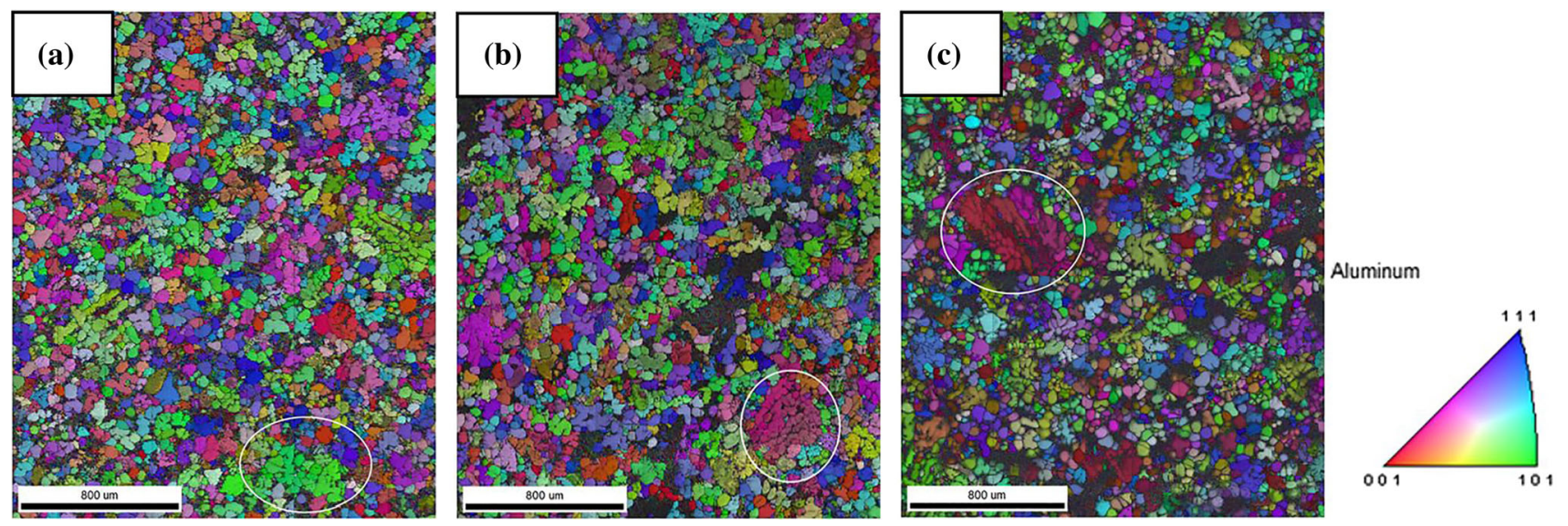

Fig. 2-EBSD maps where the individual grains are distinguished by different colours according to their crystallographic orientations; (a) base, (b) base $+\mathrm{Al}-8 \mathrm{~B}$, and $(c)$ base $+\mathrm{Al}-5 \mathrm{Ti}-1 \mathrm{~B}$. Large dendrites likely originating from the freeze-on layer are highlighted (Color figure online).

Table II. The Average Equivalent Circular Diameter and the Shape Factor Obtained for Each Casting

\begin{tabular}{lcr}
\hline Castings & Average Equivalent Circular Diameter $(\mu \mathrm{m})$ & Shape Factor \\
\hline Base & $72 \pm 19$ & $0.44 \pm 0.15$ \\
Base + Al-8B & $73 \pm 18$ & $0.46 \pm 0.16$ \\
Base + Al-5Ti-1B & $71 \pm 17$ & $0.43 \pm 0.17$ \\
\hline
\end{tabular}

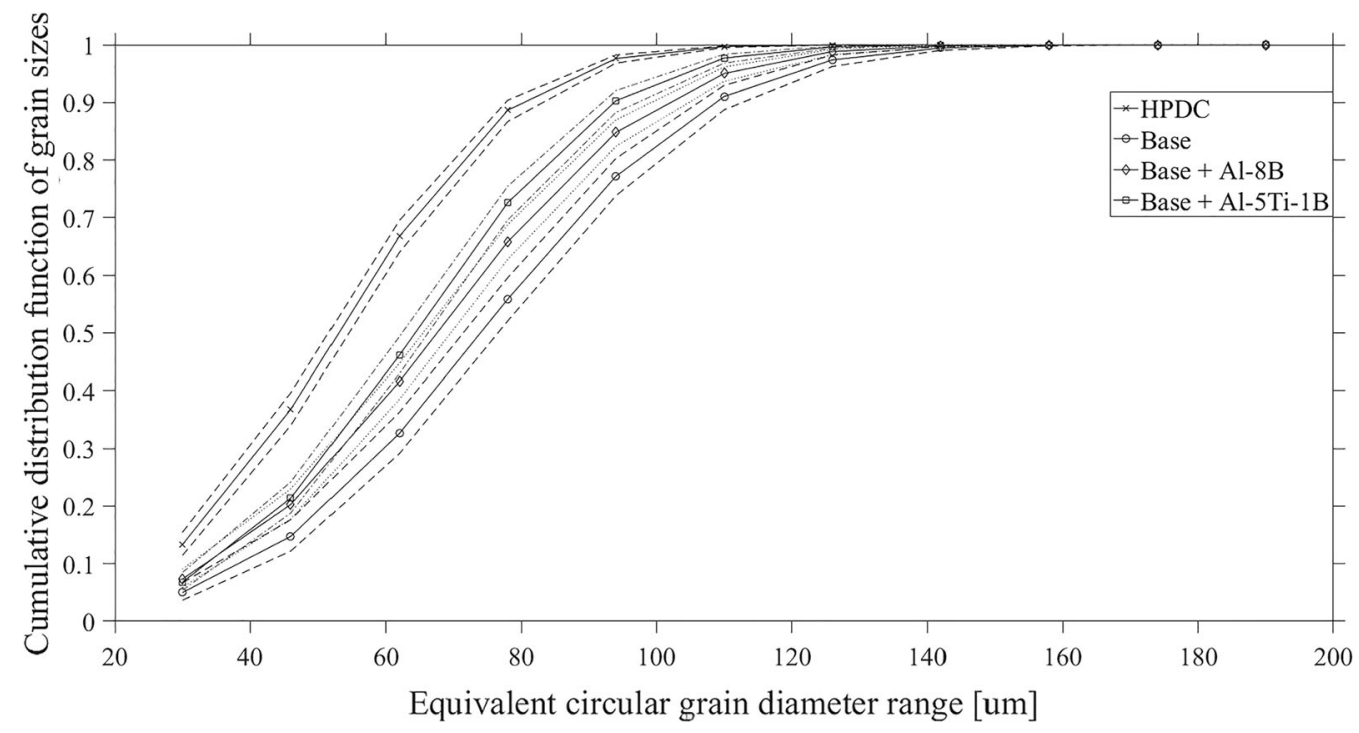

Fig. 3 - Cumulative distribution function as function of equivalent circular grain diameter. Dashed lines show 95 pct confidence interval bounds.

circular diameter $\leq 60 \mu \mathrm{m}$ compared with the unrefined casting. Therefore, grain refinement had some effect on the cast microstructures causing formation of a greater fraction of small $\alpha$-Al grains compared with the unrefined alloy.

\section{B. Solid Fraction}

Figure 4(a) shows a representative microstructure from the casting refined with Al-8B etched with Weck's reagent. The primary $\alpha_{1}-\mathrm{Al}$ globules have a dark brown core surrounded by a brighter peripheral layer. Figure 4(b)) shows the area fraction occupied by $\alpha_{1}$-Al globules, excluding and including the peripheral brighter layer. $\alpha$-Al globules with diameter $<25 \mu \mathrm{m}$ were excluded from the measurements because they have likely formed during solidification in the die-cavity. The peripheral brighter layer is assumed to have formed during solidification of the crystals in the die-cavity and the darker core is representative of the crystal size in the slurry before injection into the die-cavity. Therefore, the $\alpha_{1}-\mathrm{Al}$ area fraction excluding the peripheral brighter 

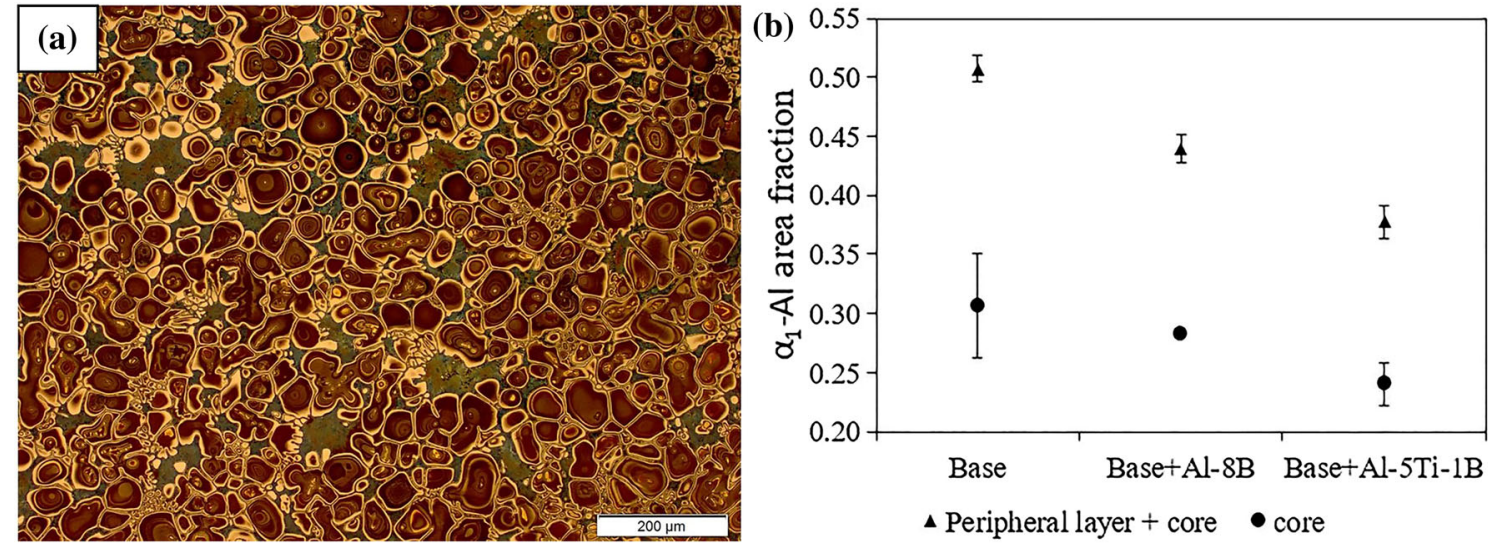

Fig. 4 - (a) Micrograph of the base + Al-8B casting etched with Weck's reagent showing $\alpha$-Al globules with a dark brown core surrounded by a brighter periphery, $(b) \alpha$-Al area fraction considering the brown core and core + periphery, as observed in (a) (Color figure online).

Table III. Silicon Concentration Measured in the Interior of the $\alpha_{1}$-Al Globules and the Corresponding Slurry Temperature and Solid Fraction Calculated in Thermocalc ${ }^{\mathrm{TM}}$ for Each Alloy

\begin{tabular}{lccc}
\hline & & \multicolumn{2}{c}{ Calculated in Thermocalc ${ }^{\mathrm{TM}}$} \\
\cline { 3 - 4 } Alloy & Silicon Content in Wt Pct & Slurry Temperature $\left({ }^{\circ} \mathrm{C}\right)$ & Solid Fraction \\
\hline Base & $0.99 \pm 0.04$ & 602 & 0.23 \\
Base + Al-8B & $1.00 \pm 0.04$ & 602 & 0.22 \\
Base + Al-5Ti-1B & $0.94 \pm 0.03$ & 605 & 0.20 \\
\hline
\end{tabular}

layer is representative of the $\alpha_{1}$-Al fraction in the slurry before injection. When the peripheral brighter layer is included, the $\alpha_{1}$-Al area fraction obtained is the $\alpha_{1}-\mathrm{Al}$ fraction when solidification is complete. The alloy refined with Al-5Ti-1B showed lesser solid fraction compared to the alloy refined with Al-8B and unrefined, Figure 4(b)). No significant difference in $\alpha_{1}-\mathrm{Al}$ fraction can be observed for the alloy refined with Al-8B compared to the unrefined alloy when only the $\alpha_{1}-\mathrm{Al}$ globule core is included. The differences are not very significant and can result from experimental measurement errors. However, when the periphery is included in the determination of $\alpha_{1}-\mathrm{Al}$ area fraction, the unrefined alloy shows a significantly larger $\alpha_{1}$-Al area fraction compared to both the grain refined alloys. The growth during solidification in the die-cavity have a great impact on the $\alpha_{1}$-Al area fraction, with the solid fraction increasing 20 pct, from 31 to 51 pct for the unrefined casting. For the refined alloys, the increase of solid fraction due to growth during solidification in the die-cavity, is smaller comparing to the unrefined alloy, 16 and 14 pct for grain refined alloys with Al-8B and Al-5Ti-1B, respectively. Figure 4(b)) shows that grain refinement influences the solidification that occurs in the die-cavity.

The silicon concentration in the interior of the $\alpha_{1}-\mathrm{Al}$ globules was measured by WDS and the results are shown in Table III. Similar to Payandeh et al. ${ }^{[17]}$ the silicon concentration in the interior of the $\alpha_{1}$-Al globules was used to calculate the corresponding solidus temperature in Thermocalc $^{\mathrm{TM}}$, for each alloy, shown in
Table III. From the solidus temperature, the $\alpha_{1}$-Al fraction in the slurry was calculated in Thermocalc ${ }^{\mathrm{TM}}$, Table III. The $\alpha_{1}$-Al solid fraction predicted in Thermocalc $^{\mathrm{TM}}$ is smaller for all the alloys in comparison to that determined from the microstructures, shown in Figure 4(b)). However, the alloy refined with Al-5Ti-1B showed the smaller difference between the Thermocalc ${ }^{\mathrm{TM}}$ prediction to that determined from micrographs of surfaces etched with Weck's reagent.

\section{Surface Microstructure}

In all alloys in this work the main difference in microstructure was found near the die wall as shown in Figure 5. The micrographs in Figure 5 show the first $1 \mathrm{~mm}$ surface layer near the die wall. A finer and more uniform microstructure is observed near the casting surface in the grain refined alloys, Figure 5(b) and (c), compared to the base alloy, Figure 5(a). In these $1 \mathrm{~mm}$ surface layers there is more primary $\alpha_{1}$-Al globules in the unrefined alloy and alloy refined with Al-8B than in the alloy refined with Al-5Ti-1B. This observation is in agreement with the observed solid fraction in Figure 4(b), where the unrefined and alloy refined with Al-8B show larger solid fraction compared to the alloy refined with Al-5Ti-1B.

The microstructure differences obtained near SSM castings surface for the different alloys suggested that solute segregation is also influenced by grain refinement. The slurry solid fraction influences the composition of 

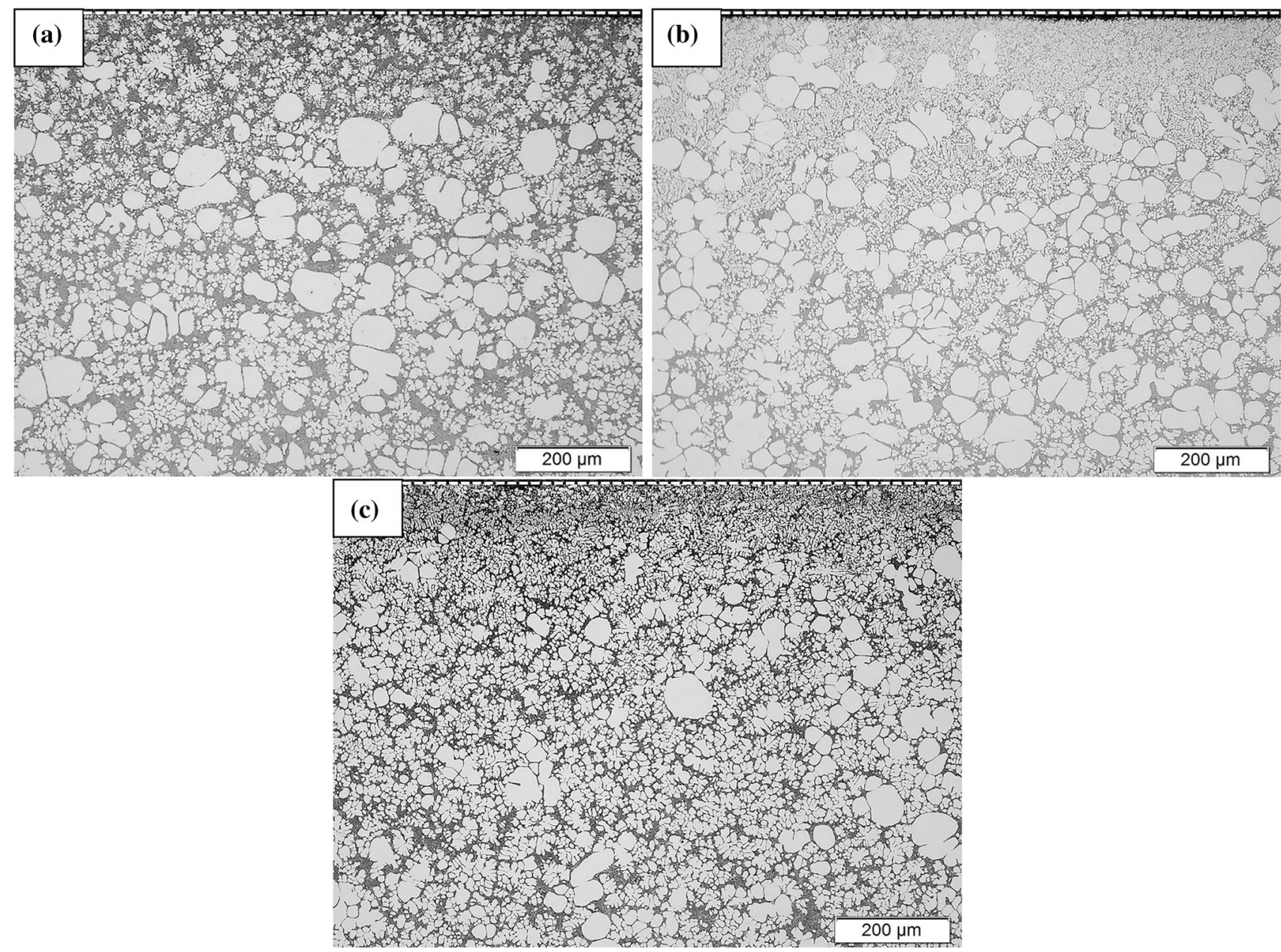

Fig. 5-Microstructures obtained near the castings surface for $(a)$ base, $(b)$ base $+\mathrm{Al}-8 \mathrm{~B},(c)$ base $+\mathrm{Al}-5 \mathrm{Ti}-1 \mathrm{~B}$ alloys. The top dashed line shows the die wall position during casting.

the remaining liquid that solidifies partly in the shot sleeve and completely in the die-cavity and, consequently, the segregation obtained in the SSM casting. The composition of the liquid injected into the die-cavity can be obtained from the mass balance:

$$
C_{\mathrm{s}} f_{\mathrm{s}}+C_{\mathrm{l}} f_{1}=C_{0}
$$

where $C_{\mathrm{s}}$ is the solute content in the interior of the $\alpha_{1}$-Al globules, Table III, $f_{\mathrm{s}}$ is the slurry solid fraction before injection into the die-cavity Figure $4(\mathrm{~b}), f_{1}=1-f_{\mathrm{s}}$ is the liquid fraction, $\mathrm{C}_{0}$ is the initial solute content of the alloy, shown in Table $\mathrm{I}$, and $C_{1}$ is the solute content in the liquid. The calculated solute contents in the liquid $\left(C_{1}\right)$ of the slurries show that the unrefined alloy has the larger solute content, $9.8 \mathrm{wt}$ pct silicon, compared to the refined alloys, 9.5 and $8.7 \mathrm{wt}$ pct silicon for the alloys refined with $\mathrm{Al}-8 \mathrm{~B}$ and $\mathrm{Al}-5 \mathrm{Ti}-1 \mathrm{~B}$, respectively, as shown in Table IV.

Figure 6(a) illustrates the slurry in the shot sleeve before injection into the die-cavity, where the $\alpha_{1}$-Al crystals are surrounded by the solute enriched liquid, with the composition shown in Table IV, for each alloy. In Figure 6(a), when the shot sleeve is at the injection position, the piston advances and forces the slurry into the die-cavity. In the die-cavity, solidification occurs with the growth of $\alpha_{1}$-Al crystals from the slurry, and nucleation and growth of in-cavity solidified crystals. Consequently, additional solute enrichment occurs in the liquid until the eutectic composition is reached. The cooling rate near the die walls is faster than in the casting center. The solidifying surface layer shrinks and the solute enriched liquid of adjacent regions can move through interdendritic channels towards the casting surface, i.e., by the inverse segregation mechanism, ${ }^{[1]}$ to compensate for solidification shrinkage and thermal contraction. The intensification pressure enhances the surface segregation. ${ }^{[27]}$ Thus, the applied intensification pressure forces the slurry to move deeper into the die-cavity and most likely increases the packing of the $\alpha$-Al crystals in the casting center. Consequently, the solute enriched liquid that previously filled the interdendritic spaces is squeezed towards the casting surface. At some point during the $\alpha_{1}$-Al crystal growth period, the flow of solute enriched liquid towards the surface region becomes difficult because the liquid flow channels become too small and tortuous. ${ }^{[28]}$ At this point, Figure 6(b), the solute content of the liquid that occupy 
the regions between the $\alpha_{1}$-Al crystals is likely to be similar to the composition of the surface segregation layer. Since the liquid flow channels towards the surface are blocked, it is possible that the solute content of the surface segregation layer remains constant until the casting is fully solidified. The $\alpha$ - $\mathrm{Al}$ crystals adjacent to the surface segregation layer continue to grow and reject more solute into the liquid until the remaining liquid reaches the eutectic composition and eutectic is formed. ${ }^{[29]}$ Figure 6(c) shows the moment when the remaining liquid reached the eutectic composition and the $\alpha_{1}$-Al crystals reached their maximum size. The brighter area surrounding the brown core of the $\alpha_{1}-\mathrm{Al}$ crystals in Figure 6(b) and in Figure 6(c) shows $\alpha_{1}-\mathrm{Al}$ crystals growth during the in-cavity solidification.

The silicon content in the liquid, Table IV, is plotted as a function of the solid fraction in the slurry before injection into the die-cavity in Figure 7(b), shown as open data markers. A direct relationship between the solute content in the liquid and the solid fraction is observed, with the silicon content increasing almost linearly with increasing $\alpha_{1}$-Al solid fraction. The greater $\alpha_{1}-\mathrm{Al}$ solid fraction obtained in the unrefined alloy resulted in more solute being rejected into the liquid

Table IV. Calculated Solute Content in the Remaining Liquid Before Injection into the Die-Cavity for the Different Alloys in Weight Percent

\begin{tabular}{lc}
\hline Alloy & Silicon Content \\
\hline Base & 9.8 \\
Base + Al-8B & 9.5 \\
Base + Al-5Ti-1B & 8.7 \\
\hline
\end{tabular}

compared to the grain refined alloys, particularly the alloy refined with Al-5Ti-1B.

The silicon content in the surface segregation layer was measured by EDS in different regions selected to avoid the $\alpha_{1}$-Al globules, as shown in Figure 7(a). The aim was to measure the silicon content of the $\alpha_{1}$-Al interdendritic regions in the surface segregation layer and these results are also shown in Figure 7(b) (closed data markers). It is assumed that the composition of these surface regions is the same as in the $\alpha_{1}$-Al interdendritic regions in the center (same liquid) at the moment that flow channels become blocked. It is observed that the silicon content in the surface region is slightly higher compared to the silicon content determined for the slurry before injection into the die-cavity. This suggests that additional solidification occurred in the die-cavity before the flow of solute enriched liquid towards the casting surface was blocked. During solidification in the die-cavity, the fraction of $\alpha_{1}-\mathrm{Al}$ crystals can be calculated by:

$$
f_{\alpha 1}^{\text {cavity }}=f_{s}^{\text {slurry }}+\Delta f_{\alpha 1}^{\text {growth }}
$$

in which $f_{\alpha 1}^{\text {eavity }}$ is the $\alpha_{1}$-Al fraction in the die-cavity, $f_{\mathrm{s}}^{\text {slurry }}$ is the solid fraction in the slurry before injection and $\Delta f_{\alpha 1}^{\text {growth }}$ is the increase in $\alpha_{1}$-Al solid fraction in the die-cavity, shown as a growth layer in Figure 6(b) and (c). In the die-cavity, the liquid of the slurry before injection, $f_{1}^{\text {slurry }}=1-f_{\mathrm{s}}^{\text {slurry }}$, can solidify by growth of the existing $\alpha_{1}$-Al crystals and nucleation and growth of in-cavity solidified crystals. However, it is likely that the growth of the existing $\alpha_{1}-\mathrm{Al}$ crystals is dominant until the surface segregation layer is formed. As described earlier, it is assumed that the solute content of the liquid in the $\alpha_{1}$-Al interdendritic region in

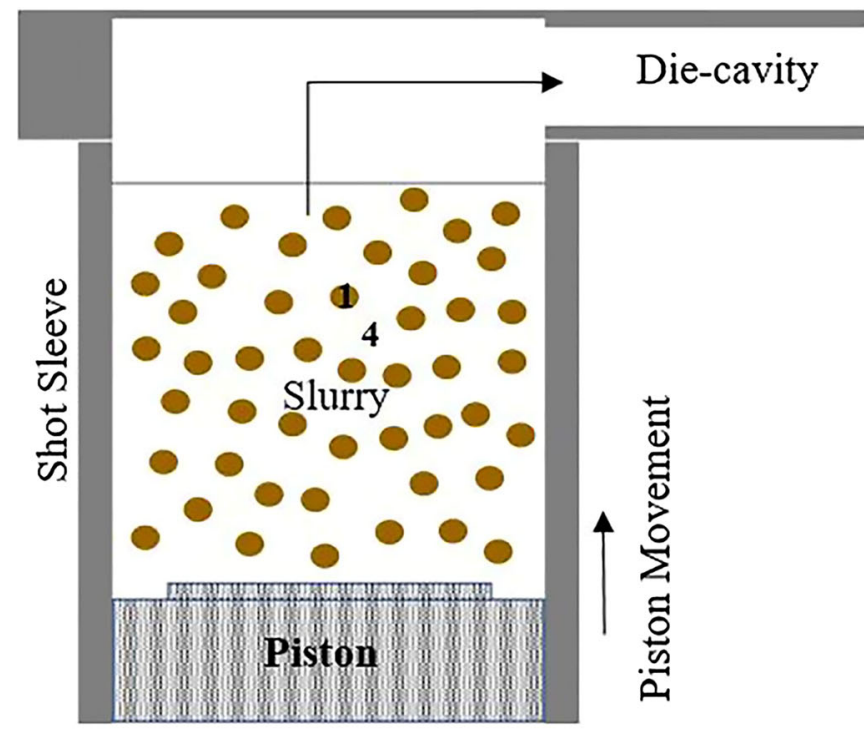

(a)
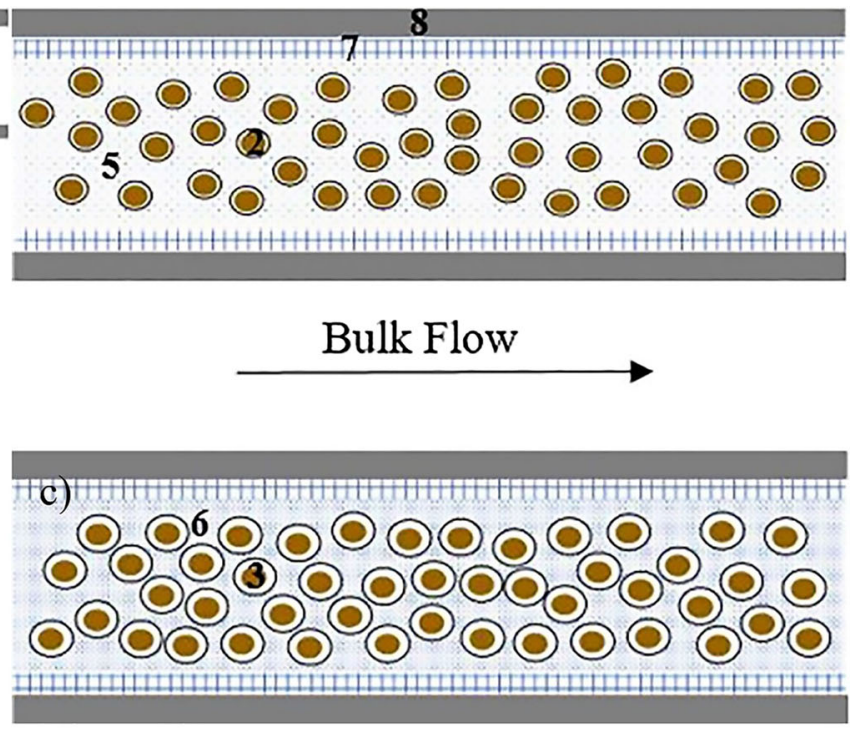

(b)

Fig. 6-Illustration of: (a) $\alpha_{1}$-Al crystals distributed in the liquid in the shot sleeve before injection into the die-cavity. (b) $\alpha_{1}$-Al crystals growth during solidification in the die-cavity and surface segregation layer. $(c) \alpha_{1}$-Al crystals growth at the end of solidification. $1-\alpha_{1}$-crystals in the shot sleeve. 2- $\alpha_{1}$-Al crystals growth and surface segregation layer. 3- $\alpha_{1}$-Al crystals growth at the end of solidification. $4-$ Solute enriched liquid. 5-Solute enriched liquid. 6-Solute enriched liquid and in-cavity solidified crystals. 7-Surface segregation layer. 8-Die wall. 


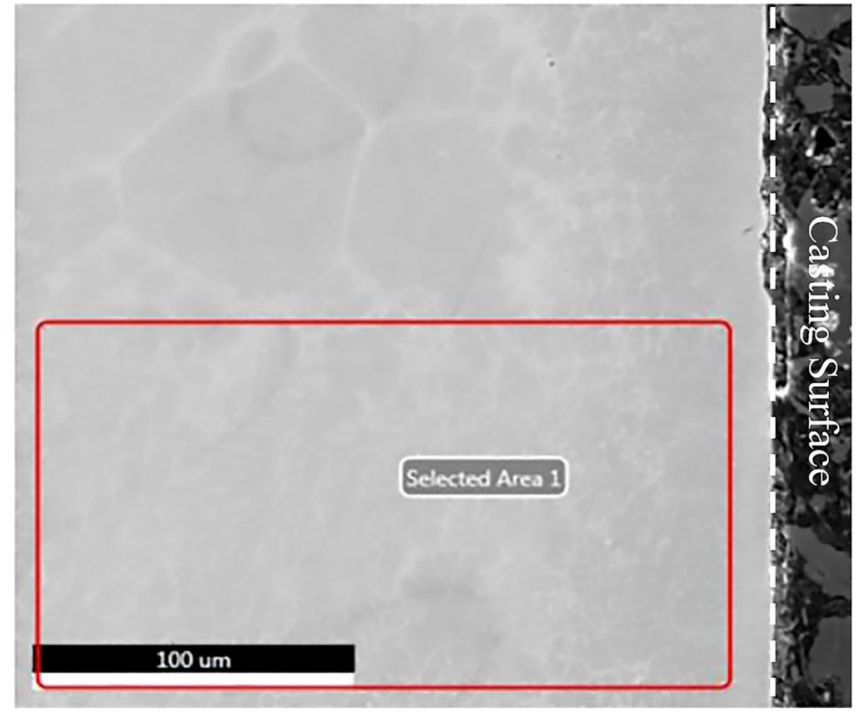

(a)

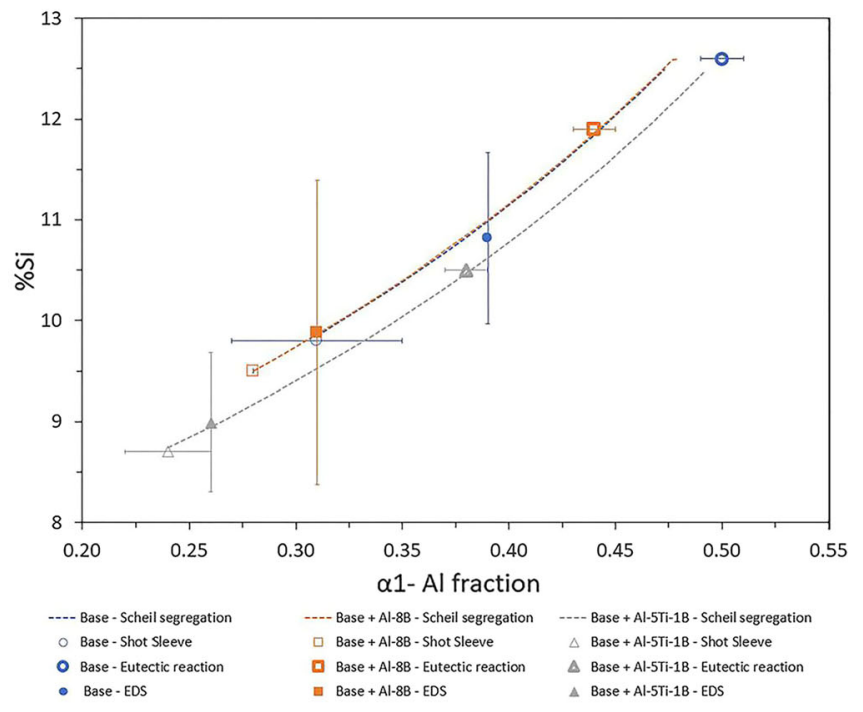

(b)

Fig. 7- (a) Region near the casting surface where silicon content was measured by EDS in the base + Al-8B alloy casting and $(b)$ evolution of silicon content in the liquid as function of $\alpha_{1}$-Al solid fraction.

the casting center is similar to the solute content of the surface segregation layer when the interdendritic channels become blocked. Therefore, the variation of silicon content as a function of solid fraction was calculated by the Scheil-Gulliver model in Thermocalc $^{\mathrm{TM}}$ for solidification of the $f_{1}^{\text {slurry }}$. The silicon content measured by EDS in the surface region was used to obtain the additional solid fraction that had formed on existing $\alpha_{1}$-Al crystals, $\Delta f_{\alpha 1}$. The $\Delta f_{\alpha 1}$, is the $f_{1}^{\text {slurry }}$ that solidified on the existing $\alpha_{1}$-Al crystals. Thus, the increase of solid fraction in the die-cavity by the growth of the $\alpha_{1}$-Al crystals, $\Delta f_{\alpha 1}^{\text {growth }}$, can be obtained by:

$$
\Delta f_{\alpha 1}^{\text {growth }}=\Delta f_{\alpha 1} \cdot f_{1}^{\text {slurry }}
$$

The $\Delta f_{\alpha 1}^{\text {growth }}$ obtained for each alloy was used to obtain the $f_{\alpha 1}^{\text {aavity }}$, Eq. [2], and plotted as closed data markers in Figure 7(b), with the respective silicon content measured by EDS.

The silicon content measured by EDS in the surface region is larger for the unrefined alloy compared to the alloy refined with $\mathrm{Al}-5 \mathrm{Ti}-1 \mathrm{~B}$, while no significant difference is found for the alloy refined with Al-8B. The addition of grain refiner, seems to move the solute content of the surface segregation region closer to the solute content of the liquid obtained before injection into the die-cavity, as shown in Figure 7(b). The difference between the slurry solid fraction before injection and the $\alpha_{1}$-Al solid fraction at which the surface segregation region is formed, is higher for the unrefined alloy compared to the refined alloys.

The $f_{\alpha 1}^{\text {eavity }}$ at which the remaining liquid reaches the eutectic composition are observed in Figure 4(b) (periphery + core) and were plotted in Figure 7(b) as open data markers. From Eq. [2], the $\Delta f_{\alpha 1}^{\text {growth }}$ can be obtained for each alloy and the $f_{1}^{\text {slurry }}$ that solidified on the existing $\alpha_{1}$-Al crystals $\left(\Delta f_{\alpha 1}\right)$ is calculated from Eq. [3]. The $\Delta f_{\alpha 1}$ can be used to obtain the silicon content of the mixture liquid + in-cavity solidified crystals from the Thermocalc $^{\mathrm{TM}}$, as described earlier. The silicon content of the region surrounded the $\alpha_{1}$-Al crystals is plotted as open data markers in Figure 7(b), with the respective $\alpha_{1}$-Al solid fraction. The comparison of silicon contents measured by EDS in the surface segregation region and that obtained when the remaining liquid reached the eutectic composition, Figure 7(b), shows that the surface segregation region most likely formed during the $\alpha_{1}$-Al crystal growth period.

For the refined alloys, the formation of a larger fraction of in-cavity solidified crystals is more likely to occur due to the lower $\alpha_{1}$-Al solid fraction compared to the unrefined alloy. This may contribute to the lower solute content found in the surface region.

\section{Primary $\alpha$-Al Globules}

The misorientation angle distribution of grain boundaries was obtained from EBSD maps and the results are shown in Figure 8. Low Angle Grain Boundaries (LAGB) are defined as boundaries with a misorientation in the range 5 to $15 \mathrm{deg}$ and High Angle Grain Boundaries (HAGB) with misorientation angles greater than 15 deg. ${ }^{[19]}$ The misorientation angles below 5 deg were not considered in order to focus the analysis on regions with significant misorientation, similar to other studies. ${ }^{[19]}$ The eutectic regions were eliminated from the results by setting a minimum grain size of $10 \mu \mathrm{m}$ and a minimum Confidence Index (CI) of 0.1. The CI provides a measure of indexing reliability and 0.1 is a typical tolerance value used. ${ }^{[30]}$

It is interesting to observe in Figure 8, that for misorientation angles between 50 to 60 degrees, the 
refined alloys show a slighter larger number fraction of grain boundaries compared to the unrefined alloy. Additionally, Figure 8 shows that the castings with grain refiner have similar evolution of grain boundaries angle distribution between 50 and 60 degrees of misorientation, while the unrefined alloy follows a different trend. However, as observed in Figure 9(a), the $\alpha$-Al grain boundaries that surround the eutectic regions are not considered in the results, due to the very low CI of these regions, and this may influence the distribution observed in Figure 8. It would be expected that a larger grain size would be obtained for the unrefined alloy compared to the refined alloys due to the slightly lower number fraction of HAGB obtained in the unrefined alloy. However, the expected larger grain size of the unrefined alloy compared to the refined alloys is likely not significant compared to the large scatter obtained in the grain size measurements for all alloys.

Figure 9(a) shows the grain boundaries identified in EBSD maps and Figure 9(b) (highlighted region) shows black lines in the interior of the $\alpha_{1}-\mathrm{Al}$ globules revealed by the Weck's reagent, as previously reported by Gao et al. to be HAGB. ${ }^{[15]}$ Additionally, in Figure 9(b), in the highlighted region, it is possible to observe what

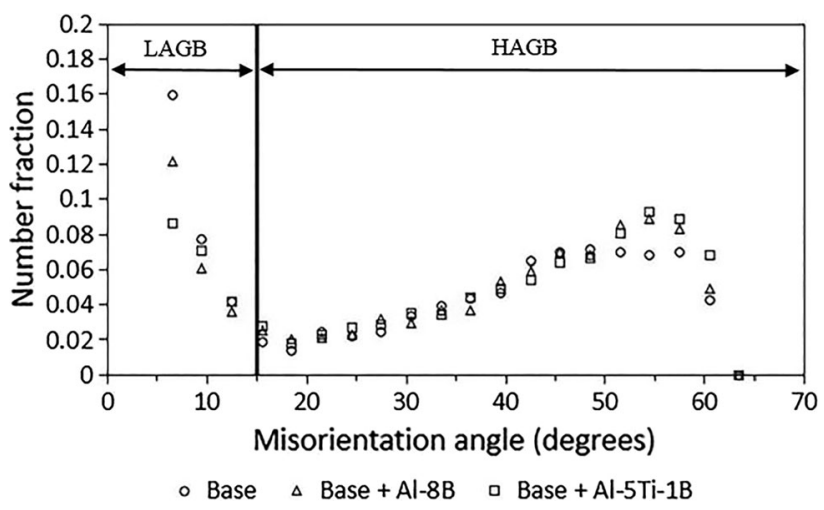

Fig. 8-Misorientation angles distribution of grain boundaries. The misorientation angles less than $5 \mathrm{deg}$ are not plotted to show just the grain boundaries between regions with significant misorientations. The vertical line separates the low angle grain boundaries $\leq 15 \mathrm{deg}$ (LAGB) from the high angle grain boundaries $>15 \mathrm{deg}$ (HAGB). seems to be liquid penetration in the $\alpha$-Al globule. Doherty et al. ${ }^{[31]}$ showed that HAGB can be obtained in bent crystals of aluminium at high temperature with the liquid wetting at the formed HAGB. The disintegration of dendrites can occur by the liquid wetting of bent crystals, ${ }^{[32]}$ when the misorientation angle exceeds $15 \mathrm{deg} .{ }^{[19]}$ More recently, disintegration of $\alpha$ - $\mathrm{Al}$ crystals by liquid wetting was observed in an $\mathrm{Al}-\mathrm{Cu}$ alloy deformed in the semi-solid range. ${ }^{[33]}$ In Figure 9(b), similar features are observed in the highlighted region, with liquid wetting (region 1, Figure 9(b)) in one of the crystals, revealing a near disintegration of the globule and an $\alpha$-Al globule where HAGB are observed, revealed by the black lines (region 2, Figure 9(b)). Therefore, this is evidence that deformation followed by liquid wetting of the HAGB can occur in the Rheome$\mathrm{tal}^{\mathrm{TM}}$ process. Disintegration of $\alpha-\mathrm{Al}$ crystals may therefore occur, most likely during the intensification pressure stage, where the crystals are more densely packed and larger stresses are transmitted between $\alpha$-Al crystals.

\section{DISCUSSION}

\section{A. Grain Formation}

The Rheometal ${ }^{\mathrm{TM}}$ slurry preparation process, like other Rheocasting processes, aims to obtain a slurry with small and globular crystals dispersed in the liquid. This slurry displays a thixotropic behavior with liquid-like properties when shear stresses are applied, e.g., during die-filling, and to cast complex shaped castings with less defects compared to coarser microstructures. The addition of grain refiners to commercial aluminium alloys is a common practice in foundries and a significant number of studies can be found in the literature focused on the Al-5Ti-1 $\mathrm{B}^{[7,34-36]}$ and $\mathrm{Al}-\mathrm{B}^{[37-39]}$ grain refiner systems. However, the effect of grain refiner addition during solidification of an alloy while stirring is applied has not received much attention in the literature. In the Rheometal ${ }^{\mathrm{TM}}$ slurry preparation process, solidification occurs while the liquid alloy is stirred by a lower enthalpy material. The cumulative distribution function

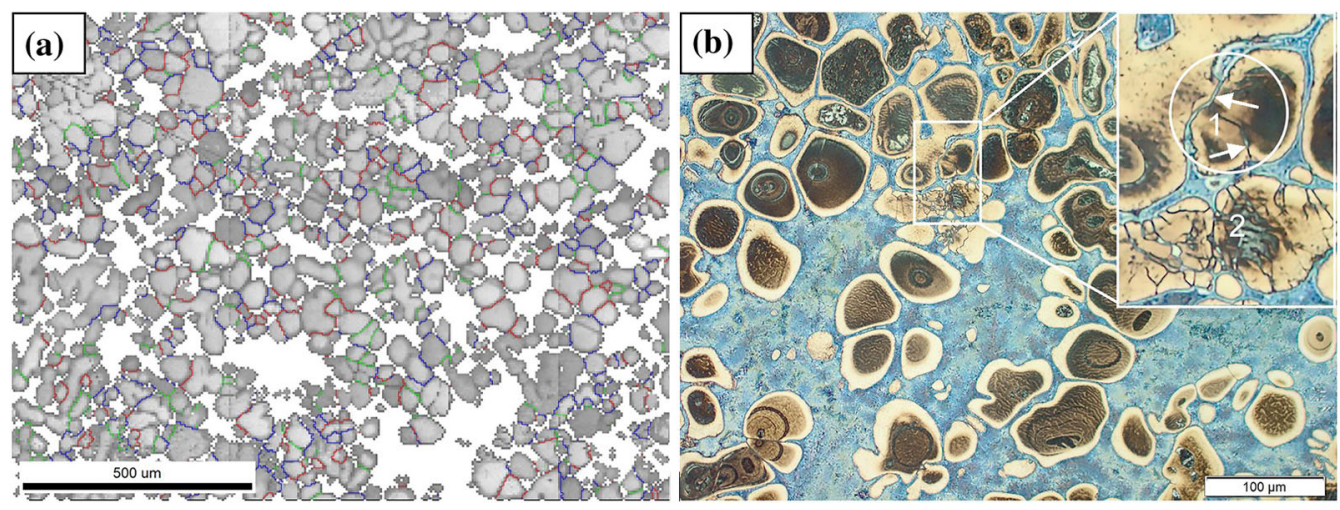

Fig. 9- (a) EBSD map from base + Al-5Ti-1B alloy casting showing LAGB (5 to 15 deg - green lines), HAGB (15 to 180 deg - blue lines, 50 to $60 \mathrm{deg}$ - red lines) and (b) micrograph from base alloy casting showing liquid wetting (region 1) and HAGB revealed with Weck's reagent (region 2) (Color figure online). 
of the grain sizes was used to evaluate the effect of grain refiners on the different alloys. The results showed slightly greater fraction of $\alpha-\mathrm{Al}$ grains with equivalent circular diameter $\leq 60 \mu \mathrm{m}$ for the refined alloys compared to the unrefined alloy, as seen in Figure 3. The primary $\alpha$-Al grain formation during the Rheometal ${ }^{\mathrm{TM}}$ slurry preparation process can be understood considering the solidification and flow conditions while the EEM is immersed and stirred in the liquid. A short time after immersion of the EEM, columnar $\alpha$-Al dendrites and equiaxed $\alpha_{1}$-Al grains were observed in microstructures of quenched EEM and slurry, respectively. ${ }^{[23]}$ Therefore, during the initial stages of Rheometal ${ }^{\mathrm{TM}}$ slurry preparation, a columnar to equiaxed growth transition occurs. A large thermal gradient is established between the $\operatorname{EEM}\left(\sim 200{ }^{\circ} \mathrm{C}\right)$ and the liquid $\left(650^{\circ} \mathrm{C}\right)$ after immersion of the EEM, resulting in a thermally undercooled region in the immediate liquid surrounding the EEM. In this region, a large amount of crystal nucleation events can occur, analogous to the "free chill crystal" nucleation mechanism proposed by Chalmers. ${ }^{[40]}$ Crystals not attached to the EEM surface are likely transported into the bulk liquid by flow caused by the rotation of the EEM. Fragmentation of the columnar $\alpha$-Al dendrites in the freeze-on layer may also occur because of the stirring and additional crystals can be mixed into the bulk liquid. ${ }^{[41]}$ The continuous stirring promotes thermal and compositional homogenization in the liquid and a rapid removal of superheat which increases the survival rate of crystals. $^{[42]}$ The final grain size is a result of the competition between nucleation rate and growth of crystals. ${ }^{[43]}$ In the initial stages of the slurry preparation process, the latent heat released by the growing crystals can be rapidly removed by the EEM and nucleation of new crystals can occur at large undercoolings. As a result, nucleation of $\alpha$-Al crystals can also occur on less potent substrates existing in the liquid in addition to the nucleant substrates introduced by the grain refiners. At the later stages of the slurry preparation, the heat extraction capacity of the EEM is reduced and the nucleation of new crystals is likely inhibited by the release of latent heat by the growth of existing $\alpha_{1}-\mathrm{Al}$ crystals in the slurry. ${ }^{[43,44]}$ That is, inhibition of nucleation occur earlier for an alloy which $\alpha$-Al crystals growing faster, resulting in greater release of latent heat and recalescence. ${ }^{[43,44]}$ The analysis of Figure 4(b) shows that the area occupied by the $\alpha_{1}$-Al crystals increases more for the unrefined alloy compared to the grain refined alloys during solidification in the die-cavity, particularly for the alloy refined with Al-5Ti-1B. That is, the $\alpha_{1}$-Al crystal growth during the slurry preparation process is likely greater for the unrefined alloy compared to the grain refined alloys. The growth restriction factor (Q) of the individual elements silicon, iron, magnesium, titanium and boron of the unrefined and refined alloys was calculated by ${ }^{[43]}$ :

$$
Q=C_{0} m(k-1)
$$

where $\mathrm{C}_{0}$ is the initial solute concentration shown in Table $\mathrm{I}, \mathrm{m}$ is the liquidus slope and $\mathrm{k}$ is the equilibrium partition coefficient of each element. The initial concentration of boron used was the amount added as $\mathrm{Al}-8 \mathrm{~B}$ grain refiner. The values of $\mathrm{m}$ and $\mathrm{k}$ for each element where obtained from the literature. ${ }^{[45,46]}$ The growth restriction effect of the various elements in each alloy was obtained by summing the growth restriction value for the different elements in the alloys. The growth restriction values obtained were 59, 56 and 66 for the base, Al-8B and Al-5Ti-1B refined alloys, respectively. The slightly smaller growth restriction values obtained for the unrefined and Al-8B refined alloys suggests a greater growth rate of $\alpha_{1}$-Al crystals formed during Rheometal $^{\mathrm{TM}}$ slurry preparation compared to the Al-5Ti-1B alloy. That is, the latent heat evolved by the growth of the nucleated crystals can inhibit the nucleation of new crystals earlier for the base alloy and alloy refined with $\mathrm{Al}-8 \mathrm{~B}$ compared to the alloy refined with Al-5Ti-1B. Therefore, the greater fraction of small grains obtained in the alloy refined with Al-5Ti-1B compared to the unrefined alloy shown in Figure 3 may be the result of the increased number of potent nucleant substrates dispersed in the liquid and the greater growth restriction factor. The alloy refined with $\mathrm{Al}-8 \mathrm{~B}$ showed a slightly larger fraction of grains up to $60 \mu \mathrm{m}$ compared to the unrefined alloy, most likely resulting from the greater number of potent nucleant substrates provided by the grain refiner. However, lesser fraction of smaller grains was obtained for the alloy refined with Al-8B compared to the alloy refined with Al-5Ti-1B, which may result from the lower growth restriction factor of the alloy refined with Al-8B.

A smaller average grain size would be expected for the refined alloys compared to the unrefined alloy resulting from the larger fraction of small grains with equivalent circular diameter $\leq 60 \mu \mathrm{m}$ obtained for the refined alloys. However, the average grain size obtained was similar for all alloys, as seen in Table II. The expected smaller grain sizes for the refined alloys are not observed in the average grain sizes values shown in Table II, most likely due to the large scatter obtained in the measurements. The solidification conditions obtained during the different stages such as slurry preparation, holding in the shot sleeve and inside the die-cavity resulted in the formation of grains with significant variation in size. ${ }^{[17]}$ Therefore, the large scatter obtained in the measurements of grain sizes of the SSM castings. In HPDC casting, most of the solidification occurs in the die-cavity at very high cooling rates which results in a fine grain microstructure. ${ }^{[2]}$ In SSM casting, a significant fraction of crystals is formed during slurry preparation that can grow large compared to the in-cavity solidified crystals. Consequently, the greater fraction of small grains shown in Figure 3 and the smaller average grain size of $44 \pm 20 \mu \mathrm{m}$ obtained for the HPDC casting compared to the SSM castings, as seen in Table II. The slight effect of the grain refiners obtained in this study can be understood considering the solidification conditions and the chemistry of the commercial base alloy used in this study. The initial thermally undercooled region near the EEM and dendrite fragments originating from the freeze-on layer may decrease the effect of grain refinement as observed in Reference 5 for an electromagnetically stirred liquid alloy. In the New Rheocasting 
process the grain refiner effect decreased as the pouring temperature was reduced because of the large thermal undercooling obtained when the liquid contacts the cold wall of the cup. ${ }^{[7]}$ The disintegration of the freeze-on layer and EEM result in additional crystals introduced into the liquid as observed by Payandeh et al. ${ }^{[23]}$ which are unlikely affected by the grain refiner particles in the liquid. The addition of Al-5Ti-1B grain refiner introduces soluble $\mathrm{TiAl}_{3}$ and insoluble $\mathrm{TiB}_{2}$ particles into the liquid. ${ }^{[47,48]}$ Many studies reported the poisoning effect of silicon on the $\mathrm{TiB}_{2}$ particles that reduced the grain refinement efficiency. ${ }^{[34,47]}$ Therefore, the poisoning effect of silicon on $\mathrm{TiB}_{2}$ can explain the low effect of $\mathrm{Al}-5 \mathrm{Ti}-1 \mathrm{~B}$ grain refiner in this study. The $\mathrm{AlB}_{2}$ particles introduced by Al-B master alloys into liquid aluminium showed enhanced grain refining effect in hypoeutectic Al-Si foundry alloys compared to Al-5Ti-1B master alloy in different studies. ${ }^{[38,39,49]}$ Chen et al.$^{[39]}$ proposed that the formation of a $\mathrm{SiB}_{6}$ layer at the interface between $\mathrm{AlB}_{2}$ and $\mathrm{Al}$ can significantly improve the nucleation potency of the $\mathrm{AlB}_{2}$ particles. Alamdari et $a l .{ }^{[37]}$ studied the heterogeneous nucleation mechanism of boron particles in aluminium by addition of ultrafine boron particles in pure aluminium and Al-Ti melts. It was found that boron dissolves very rapidly in pure aluminium. However, in aluminium liquid with solute titanium the boron dissolution is inhibited by the formation of a thin layer of $\mathrm{TiB}_{2}$ on boron particles which can act as nucleants for $\alpha-\mathrm{Al}$. Birol ${ }^{[47]}$ found that the grain refinement effect of Al-B is poor in commercial Al-7Si-Mg alloys containing 0.04 to $0.1 \mathrm{wt}$ pet titanium due to the formation of $\mathrm{TiB}_{2}$ particles which are poisoned by silicon. ${ }^{[47]}$ In the present work a commercial Al-7Si-0.3Mg base alloy was used that contained a significant amount of titanium, as is typical for commercial aluminium alloys. ${ }^{[47]}$ This titanium could interact with the boron introduced into the liquid by the $\mathrm{Al}-8 \mathrm{~B}$ grain refiner forming $\mathrm{TiB}_{2}$ particles. Consequently, the grain refinement effectiveness of Al-8B inoculant was most likely influenced by the formation of $\mathrm{TiB}_{2}$ particles which are poisoned by silicon. Additionally, the interaction of strontium in the base alloy with boron could also negatively affect the grain refinement of the primary $\alpha-\mathrm{Al} .^{48,50]}$

Another potential negative influence on grain refiner efficiency could be the stirring effect on agglomeration of nucleant substrates. Wang et al. ${ }^{[38]}$ found that mechanical stirring applied during Al-B master alloys production resulted in large $\mathrm{AlB}_{2}$ agglomerates with increased settling tendency in molten aluminium. That is, a loss of the inoculant refinement potency can occur by agglomeration and settling of substrates as a result of increased nucleant particles interactions due to stirring. Schaffer and Dahle ${ }^{[51]}$ suggested that agglomeration has a significant effect in determining the rate at which the loss of refinement occurs due to nucleant substrates settling, which was much faster than predicted by Stokes law. The much shorter stirring time applied in this work (18 seconds) compared to other studies ${ }^{[38]}$ could suggest that stirring would have a smaller effect on agglomeration of substrates. However, in this study stirring was applied under non-isothermal conditions during solidification of refined alloys, while most other studies focus on isothermal stirring of a liquid with nucleant substrates. ${ }^{[38,51,52]}$ In a semi-solid material, the volume of liquid is comparatively less than in the fully liquid condition. Therefore, the probability of particle interactions (e.g., agglomeration) can be expected to be higher and gradually increase with decreased liquid volume. Consequently, further studies are required to understand the effect of stirring on the refinement efficiency of nucleant substrates.

After the Rheometal ${ }^{\mathrm{TM}}$ slurry preparation process is complete, the mixture of $\alpha_{1}$-Al crystals and solute enriched liquid obtained is poured into the shot sleeve. During pouring of the mixture into the shot sleeve, the formation of new $\alpha$-Al crystals is restricted to the thermal undercooled regions near the shot sleeve wall and plunger. ${ }^{[22]}$ The addition of grain refiner to HPDC titanium free A356 alloy resulted in larger fraction of crystals solidified in the shot sleeve and finer grain size compared to the unrefined alloy. ${ }^{[22]}$ However, there is significant differences between HPDC and SSM casting that are important to analyze. First, in HPDC a superheated liquid is poured in the shot sleeve while in SSM casting a slurry is poured. As a result, the latent heat released by the growth of the slurry $\alpha_{1}$-Al crystals in the shot sleeve is likely more significant than that released by the growth of the lower fraction of crystals formed in the shot sleeve in HPDC. Therefore, the nucleation of new crystals between the pre-existing crystals during slurry holding in the shot sleeve may be inhibited for SSM castings. Secondly, the silicon content of the existing liquid of the slurry poured into the shot sleeve is larger in SSM casting compared to HPDC of the same alloy. Consequently, the poisoning effect of silicon of the $\mathrm{TiB}_{2}$ particles dispersed in the liquid can be increased and reduce the number of active substrates dispersed in the liquid of the slurry.

In the die-cavity, the solidification occurs at very large cooling rates and for this reason the addition of grain refiner is considered unnecessary to obtain a fine microstructure. ${ }^{[22]}$ Additionally, increased silicon content of the existing liquid of the slurry, as seen in Table IV, can reduce the effect of $\mathrm{TiB}_{2}$ substrates dispersed in the refined alloys. The growth of $\alpha_{1}-\mathrm{Al}$ crystals in the die-cavity was smaller for the refined alloys compared to the unrefined alloy, as shown in Figure 4(b), particularly for the alloy refined with Al-5Ti-1B. The lesser growth and solid fraction obtained for the alloy refined with Al-5Ti-1B can result in larger fraction of in-cavity solidified crystals compared to the other alloys and consequently, finer microstructure obtained, as shown in Figure 5(c). The potent substrates dispersed in the liquid in the grain refined alloys can assist the nucleation of in-cavity solidified crystals regardless of the poisoning effect of silicon enriched liquid.

\section{B. Solid Fraction}

The greater $\alpha_{1}$-Al fraction observed in Figure 4(b) for the unrefined alloy and $\mathrm{Al}-8 \mathrm{~B}$ refined alloy comparing to the alloy refined with Al-5Ti-1B can result from the 
lower growth restriction factor of the former alloys. As discussed earlier, the growth of $\alpha_{1}-\mathrm{Al}$ crystals during solidification in the die-cavity is larger for the unrefined alloy compared to the unrefined alloys, particularly for the alloy refined with Al-5Ti-1B. The slightly smaller growth restriction values obtained for the unrefined and $\mathrm{Al}-8 \mathrm{~B}$ refined alloys compared to the Al-5Ti-1B refined alloy suggests that a greater growth can occur for the slurry $\alpha_{1}-\mathrm{Al}$ crystals of the former alloys. Therefore, the larger slurry $\alpha_{1}-\mathrm{Al}$ area fraction (Figure 4(b)) obtained for these alloys compared to the alloy refined with $\mathrm{Al}-5 \mathrm{Ti}-1 \mathrm{~B}$ can result from the growth restriction factor. However, it would be expected that a smaller grain size would be obtained for the alloy refined with Al-5Ti-1B, which is contrary to the results obtained. The effect of grain refiner addition on $\alpha_{1}$-Al crystal fraction obtained during stirring needs further work as the solid fraction of the slurry influences significantly the filling and feeding behavior during SSM casting.

\section{Surface Segregation}

A finer and more uniform microstructure was obtained near casting surface for the refined castings compared to the unrefined, as seen in Figure 5. Additionally, the silicon content obtained in the surface segregation layer was lower for the alloy refined with Al-5Ti-1B $(9.0 \pm 0.7 \mathrm{wt}$ pct $)$ compared to the unrefined alloy (10.8 $\pm 0.8 \mathrm{wt} \mathrm{pct})$. Moreover, lesser solid fraction was obtained in the alloy refined with Al-5Ti-1B, $0.24 \pm 0.02$ compared to the unrefined alloy, $0.31 \pm 0.04$. Typically, in HPDC a mixture of externally solidified crystals and liquid is injected into the die-cavity. ${ }^{[19]}$ Laukli et al. ${ }^{[22]}$ obtained greater silicon content near surface of HPDC A356 alloy castings surface with larger fraction of externally solidified crystals compared to castings with lesser fraction of externally solidified crystals. During die-cavity filling, the externally solidified crystals migrate to the central region of the casting and few are observed near casting surface. ${ }^{[18]}$ Similar behavior is expected from the $\alpha_{1}-\mathrm{Al}$ crystals formed during the Rheometal ${ }^{\mathrm{TM}}$ slurry preparation during die-cavity filling. Near the casting surface the existing solute enriched liquid solidifies into very small $\alpha-\mathrm{Al}$ dendrites and eutectic. In addition to the concentration of the externally solidified crystals in the casting center, inverse segregation can contribute to the surface segregation layer formation. ${ }^{[1]}$ The fraction of eutectic formed near the casting surface is influenced by the composition of the existing liquid in the die-cavity, which depends on the fraction of externally solidified crystals formed during slurry preparation and their growth in the die-cavity. Therefore, it is reasonable to assume that the finer and more uniform surface microstructure obtained in the alloy refined with Al-5Ti-1B is due to the lesser solid fraction obtained and the greater growth restriction factor of the alloy. These factors can promote the formation of more in-cavity solidified crystals near the casting surface. The greater fraction of small in-cavity solidified crystals formed in the alloy refined with $\mathrm{Al}-5 \mathrm{Ti}-1 \mathrm{~B}$ reduce the permeability of the interdendritic regions and consequently can reduce the effect of inverse segregation.

\section{CONCLUSIONS}

In the current study, grain refined and unrefined semi-solid Al-7Si-0.3Mg castings were produced using the Rheometal ${ }^{\mathrm{TM}}$ process. The effect of grain refiner addition on primary $\alpha$-Al grain size, shape factor and solid fraction was studied. The $\alpha$-Al grain formation during the Rheometal ${ }^{\mathrm{TM}}$ process can be understood considering the solidification and flow conditions existing in the different stages of the process; slurry preparation, holding in the shot sleeve and solidification in the die-cavity. During slurry preparation a large amount of crystals is nucleated either on or near the EEM wall due to thermal undercooling. The rapid extraction of superheat by the EEM increases the survival rate of crystals dispersed into the bulk liquid. The potent nucleant substrates introduced in the liquid by the grain refiners can enhance the nucleation events that occur near the EEM wall during slurry preparation as suggested by the greater fraction of smaller $\alpha$-Al grains obtained in the refined alloys. The greatest growth restriction factor of the alloy refined with Al-5Ti-1B can result in a slower growth of $\alpha$-Al crystals and, as a result, a slower release of latent heat. Consequently, more nucleation events can occur and therefore the largest fraction of smaller $\alpha$-Al grains was obtained in the alloy refined with Al-5Ti-1B. However, the larger fraction of smaller grains obtained for the grain refined alloys do not result in a smaller average grain size. The large thermal undercooling near the EEM walls, agglomeration of nucleants during stirring, the continuous silicon enrichment of the liquid and the titanium content of the base alloy can explain the rather small effect of grain refiners in this study. Further work is required to understand the effect of stirring on grain refinement efficiency.

The smaller growth restriction factors of the base alloy and alloy refined with $\mathrm{Al}-8 \mathrm{~B}$ resulted in the more growth of $\alpha$-Al crystals in the die-cavity. The smaller and more uniform surface microstructure obtained in the refined alloys are most likely the result of the lesser solid fraction obtained compared to the base alloy. Additionally, the larger fraction of smaller grains formed in the refined alloys compared to the base alloy reduce the permeability of the interdendritic regions and consequently reduce the effect of inverse segregation. The lower solid fraction and the reduction of inverse segregation effect can explain the observation of surface segregation in the alloy refined with Al-5Ti-1B.

Evidence of liquid penetration in some of the $\alpha-\mathrm{Al}$ globules was observed, which suggests that disintegration of $\alpha$-Al globules due to liquid wetting of the deformed crystals can occur during the Rheometal ${ }^{\mathrm{TM}}$ casting process. The grain refined castings showed slightly higher fraction of grain boundaries with misorientation angles between 50 and $60 \mathrm{deg}$ in the $\alpha$-Al globules compared to the non-grain refined casting, which may suggest that more severe plastic deformation occurs in $\alpha$-Al globules in the grain refined castings. 


\section{ACKNOWLEDGMENTS}

This work was funded by VINNOVA under the FatSS project (Dnr 2014-05096) and part of the LIGHTER programme. The authors are also grateful for the support by Volvo Lastvagnar AB, COMPtech $\mathrm{AB}$, Fueltech $\mathrm{AB}$ in the project.

\section{OPEN ACCESS}

This article is distributed under the terms of the Creative Commons Attribution 4.0 International License (http://creativecommons.org/licenses/by/4.0/), which permits unrestricted use, distribution, and reproduction in any medium, provided you give appropriate credit to the original author(s) and the source, provide a link to the Creative Commons license, and indicate if changes were made.

\section{REFERENCES}

1. J. Wannasin, R. Canyook, S. Wisutmethangoon, and M.C. Flemings: Acta Mater., 2013, vol. 61, pp. 3897-3903.

2. M.A. Easton, M. Qian, A. Prasad, and D.H. StJohn: Curr. Opin. Solid State Mater. Sci., 2015, vol. 20, pp. 13-24.

3. S. Nafisi and R. Ghomashchi: J. Mater. Process. Technol., 2006, vol. 174, pp. 371-83.

4. K.T. Kashyap and T. Chandrashekar: Bull. Mater. Sci., 2001, vol. 24, pp. 345-53.

5. Z. Yang, J.W. Bae, and C.G. Kang: Solid State Phenom., 2006, vols. 116-117, pp. 344-49.

6. A. Sharma: Metall. Ital., 2013, vol. 105, pp. 53-58

7. M.A. Easton, H. Kaufmann, and W. Fragner: Mater. Sci. Eng. A, 2006, vol. 420, pp. 135-43.

8. S. Otarawanna, C.M. Gourlay, H.I. Laukli, and A.K. Dahle: Mater. Chem. Phys., 2011, vol. 130, pp. 251-58.

9. R. Nadella, D.G. Eskin, Q. Du, and L. Katgerman: Prog. Mater. Sci., 2008, vol. 53, pp. 421-80.

10. G. Govender and H. Möller: Solid State Phenom., 2008, vol. 143, pp. 433-38.

11. C.M. Gourlay, H.I. Laukli, and A.K. Dahle: Metall. Mater. Trans. A, 2007, vol. 38, pp. 1833-44.

12. O. Granath, M. Wessén, and H. Cao: Int. J. Cast Met. Res., 2008, vol. 21 , pp. 349-56.

13. ASTM E562-11: ASTM Int., 2011, pp. 1-7.

14. L. Gao, Y. Harada, and S. Kumai: Mater. Charact., 2015, vol. 107 , pp. 434-52.

15. L. Gao, Y. Harada, and S. Kumai: Mater. Charact., 2015, vol. 107 , pp. 426-33.

16. X.G. Hu, Q. Zhu, H.V. Atkinson, H.X. Lu, F. Zhang, H.B. Dong, and Y.L. Kang: Acta Mater., 2017, vol. 124, pp. 410-20.

17. M. Payandeh, A.E.W. Jarfors, and M. Wessén: Metall. Mater. Trans. A Phys. Metall. Mater. Sci., 2016, vol. 47A, pp. 1215-28.

18. H.I. Laukli, C.M. Gourlay, and A.K. Dahle: Metall. Mater. Trans. A Phys. Metall. Mater. Sci., 2005, vol. 36A, pp. 805-18.

19. S. Otarawanna, C.M. Gourlay, H.I. Laukli, and A.K. Dahle: Acta Mater., 2010, vol. 58, pp. 261-71.
20. M. Payandeh, A.E.W. Jarfors, and M. Wessén: Solid State Phenom., 2013, vol. 192, pp. 392-97.

21. M. Hitchcock, Y. Wang, and Z. Fan: Acta Mater., 2007, vol. 55, pp. $1589-98$

22. H.I. Laukli, L. Arnberg, and O. Lohne: Int. J. Cast Met. Res., 2005, vol. 18 , pp. 65-72.

23. M. Payandeh, M.H. Sabzevar, A.E.W. Jarfors, and M. Wessén: Metall. Mater. Trans. B, 2017, vol. 47B, pp. 2836-48.

24. M. Yan and W. Luo: Mater. Chem. Phys., 2007, vol. 104, pp. $267-70$.

25. A. Sharma, R. Gupta, and L. Ratke: in Materials Science and Technology Conference and Exhibition 2014, MS and T 2014, 2014, vol. 2, pp. 1243-51.

26. M.N. Laila Masrur, A.S. Anasyida, and H. Zuhailawati: Trans Tech Publications Ltd, 2014, vol. 1024, pp. 251-54.

27. S.G. Lee, G.R. Patel, and A.M. Gokhale: Mater. Charact., 2005, vol. 55, pp. 219-24

28. C.M. Gourlay, A.K. Dahle, and H.I. Laukli: Metall. Mater. Trans. A, 2004, vol. 35A, pp. 2881-91.

29. L. Bolzoni and N. Hari Babu: Appl. Mater. Today, 2016, vol. 5, pp. 255-59.

30. A.K. Dahle, D.H. St John, P. Attavanich, and P. Taopetch: Mater. Sci. Forum, 2000, vols. 331-337, pp. 271-76.

31. R.D. Doherty, H.-I. Lee, and E.A. Feest: Mater. Sci. Eng., 1984, vol. 65 , pp. $181-89$

32. S. Ji: J. Mater. Sci., 2003, vol. 38, pp. 1559-64.

33. S. Karagadde, P.D. Lee, B. Cai, J.L. Fife, M.A. Azeem, K.M. Kareh, C. Puncreobutr, D. Tsivoulas, T. Connolley, and R.C. Atwood: Nat. Commun., 2015, vol. 6, p. 8300.

34. Y.C. Lee, A.K. Dahle, D.H. StJohn, and J.E.C. Hutt: Mater. Sci. Eng. A, 1999, vol. 259, pp. 43-52.

35. D. Qiu, J.A. Taylor, M.X. Zhang, and P.M. Kelly: Acta Mater., 2007, vol. 55, pp. $1447-56$.

36. M. Riestra, E. Ghassemali, T. Bogdanoff, and S. Seifeddine: Mater. Sci. Eng. A, 2017, vol. 703, pp. 270-79.

37. H.D. Alamdari, D. Dubé, and P. Tessier: Metall. Mater. Trans. A Phys. Metall. Mater. Sci., 2013, vol. 44, pp. 388-94.

38. T. Wang, Z. Chen, H. Fu, L. Gao, and T. Li: Mater. Sci. Eng. A, 2012, vol. 549, pp. 136-43.

39. Z. Chen, H. Kang, G. Fan, J. Li, Y. Lu, J. Jie, Y. Zhang, T. Li, X. Jian, and T. Wang: Acta Mater., 2016, vol. 120, pp. 168-78.

40. B. Chalmers: J. Aust. Inst. Met., 1963, vol. 8, pp. 255-63.

41. S. Ji and Z. Fan: Metall. Mater. Trans. A, 2002, vol. 33A, pp. 3511-20.

42. W.D. Griffiths and D.G. McCartney: Mater. Sci. Eng. A, 1996, vol. 216, pp. 47-60.

43. I. Maxwell and A. Hellawell: Acta Metall., 1975, vol. 23 (229-3), p. 7 .

44. A.L. Greer, A.M. Bunn, A. Tronche, P.V. Evans, and D.J. Bristow: Acta Mater., 2000, vol. 48, pp. 2823-35.

45. M. Easton and D. St John: Metall. Mater. Trans. A, 2005, vol. 36A, pp. 1911-20.

46. D.G. McCartney: Int. Mater. Rev., 1989, vol. 34, pp. 247-60.

47. Y. Birol: Mater. Sci. Technol., 2012, vol. 28, pp. 481-86.

48. L. Lu and A.K. Dahle: Light Met., 2006, vol. 2006, pp. 807-12.

49. J. Li, G. Yang, F.S. Hage, Z. Chen, T. Wang, Q.M. Ramasse, and P. Schumacher: Mater. Charact., 2017, vol. 128, pp. 7-13.

50. K. Nogita, S.D. Mcdonald, and A.K. Dahle: Mater. Trans., 2003, vol. 44, pp. 692-95.

51. P.L. Schaffer and A.K. Dahle: Mater. Sci. Eng. A, 2005, vols. 413-414, pp. 373-78.

52. K. Sukumaran, B. Pai, and M. Chakraborty: Mater. Sci. Eng. A, 2004 , vol. 369 , pp. $275-83$. 